\title{
ON THE $S_{2}$-FICATION OF SOME TORIC VARIETIES
}

\author{
MARCEL MORALES \\ Université de Grenoble I, Institut Fourier, URA 188, B.P.74, \\ 38402 Saint-Martin D'Hères Cedex, \\ and IUFM de Lyon, 5 rue Anselme, \\ 69317 Lyon Cedex (FRANCE)
}

First version in 2004, revised version march 2006

ABSTRACT. In this paper we prove:

1. Some results on the Cohen-Macaulayness of the canonical module.

2. We study the $S_{2}$-fication of rings which are quotients by lattices ideals.

3. Given a simplicial lattice ideal of codimension two I, its Macaulayfication is given explicitly from a system of generators of $I$.

\section{Introduction}

Let $X$ be an algebraic variety, the set of points where $X$ is not Cohen-Macaulay is the Non-CohenMacaulay locus, this set was study in 1. Macaulayfication is an analogous operation to resolution of singularities and was considered in [7] where the main theorem of Macaulayfication is given.

For any affine semigroup (without torsion) $S \subset \mathbb{N}^{n}$ let $G(S)$ be the subgroup of $\mathbb{Z}^{n}$ generated by $S$ and $\bar{S}$ be the saturation of $S$ inside $G(S)$, that is

$$
\bar{S}=\{m \in G(S): r m \in S \text { for some } r \in \mathbb{N}\},
$$

it is well known that the normalization of the semigroup ring $K[S]$ is given by $K[\bar{S}]$ and Hochster proved in $[\underline{6}$ that $K[\bar{S}]$ is always a Cohen-Macaulay ring. We have an exact sequence:

$$
0 \longrightarrow K[S] \longrightarrow K[\bar{S}] \longrightarrow K[\bar{S} \backslash S] \longrightarrow 0,
$$

and $K[\bar{S}]$ is a Cohen-Macaulay ring containing $K[S]$, with the same ring of fractions. In general, the support of $K[\bar{S} \backslash S]$ does not coincide with the Non Cohen-Macaulay locus of $K[S]$ because $\bar{S}$ is too big. Our problem consist to look for a "minimal" subsemigroup $\tilde{S} \subset \bar{S}$ containing $S$ such that $K[\tilde{S}]$ is a Cohen-Macaulay ring. In [5] and [10] the authors consider a semigroup $S^{\prime} \subset \bar{S}$ which contains $S$ such that we have an exact sequence:

$$
0 \longrightarrow K[S] \longrightarrow K\left[S^{\prime}\right] \longrightarrow K\left[S^{\prime} \backslash S\right] \longrightarrow 0,
$$

and $\operatorname{dim} K\left[S^{\prime} \backslash S\right] \leq n-2 . K\left[S^{\prime}\right]$ is the $S_{2}$-fication of $K[S]$. When $K\left[S^{\prime}\right]$ is a Cohen-Macaulay ring, the support of $K[\bar{S} \backslash S]$ coincide with the Non Cohen-Macaulay locus of $K[S]$. This is the case notably when $S$ is a simplicial semigroup. The purpose of this paper is to give effective methods to compute the $S_{2}$-fication for a class of toric varieties. In the first part of this paper we consider the $S_{2}$-fication and give some general results on the Cohen Macaulayness of the canonical module, one 
of them extends and improves Proposition 2.5 of $[4$. We also extend and improve to the lattice case the above results from [5] and [10, given shorter proofs.

In the second part we consider a codimension two simplicial toric ring $K[S]$, and describe the Macaulayfication of this ring in terms of the system of generators of its ideal of definition as described in [8], this ideal can be computed by an effective algorithm which works in polynomial time at very low cost. This is also implemented in my software codim2simplicial, which computes the generators of a simplicial codimension 2 lattice ideal without using Groebner basis.

During the meeting Current trends in Commutative Algebra held in Levico, Italy, in June 2002, I have submitted to Peter Schenzel, the problem developed in this paper in sections two to four, then we have started a joint work on this subject during more than one year. Peter Schenzel got a proof using spectral sequences and decided to publish by himself in [13]. My proof developed here is completely different and elementary, it is a complement to Schenzel's proof.

\section{Known results on local cohomology}

The following results are well known [1], 12] section 1.2. All this results are also true for graded ring and modules.

Let $(R, Q)$ be a Gorenstein local ring of dimension $n$, let $(A, \mathrm{~m})$ be a factor ring of $R$ and $M$ a finitely generated $A$-module of dimension $d$.

We recall the local duality's theorem:

Theorem 1 We have an isomorphism :

$$
H_{\mathrm{m}}^{i}(M) \simeq H_{Q}^{i}(M) \simeq \operatorname{Hom}_{R}\left(\operatorname{Ext}_{R}^{n-i}(M, R), E(R / Q)\right)
$$

We denote by $D^{i}(M)$ the finitely generated $R$-module $\operatorname{Ext}_{R}^{n-i}(M, R)$, and we set by $K_{M}=D^{d}(M)$ the canonical module. We recall some of their properties:

1. For any exact sequence

$$
0 \longrightarrow M^{\prime} \longrightarrow M \longrightarrow M^{\prime \prime} \longrightarrow 0
$$

we have a long exact sequence:

$$
\ldots \longrightarrow D^{i}\left(M^{\prime \prime}\right) \longrightarrow D^{i}(M) \longrightarrow D^{i}\left(M^{\prime}\right) \longrightarrow D^{i-1}\left(M^{\prime \prime}\right) \longrightarrow D^{i-1}(M) \longrightarrow D^{i-1}\left(M^{\prime}\right) \longrightarrow
$$

2. $D^{i}(M)=0$ for either $i>d$ or $i<0, D^{d}(M)$ has dimension $d$. Moreover depth $D^{d}(M) \geq \min \{d, 2\}, D^{d}(M)$ satisfies the condition $S_{2}$ when $d \geq 2$, and if $M$ is CohenMacaulay then so is $D^{d}(M)$.

3. For all $P \in \operatorname{Supp} M$ we have $\left(D^{d}(M)\right)_{P}=D^{d}\left(M_{P}\right)$.

4. $\operatorname{dim} D^{i}(M) \leq i$ for all $0 \leq i<d$. Suppose in addition that $M$ is equidimensional. Then $M$ satisfies the condition $S_{k}$ if and only if $\operatorname{dim} D^{i}(M) \leq i-k$ for all $0 \leq i<d$.

5. If $M$ is unmixed and $d \geq 2$, then we have an exact sequence :

$$
0 \longrightarrow M \longrightarrow D^{d}\left(D^{d}(M)\right) \longrightarrow N \longrightarrow 0
$$

where $\operatorname{dim} N \leq \operatorname{dim} M-2$. Moreover $M$ satisfies the condition $S_{2}$ if and only if $M$ is isomorphic to $D^{d}\left(D^{d}(M)\right)$. 


\section{One result on the canonical module}

Theorem 2 Let $(A, \mathrm{~m})$ be a factor ring of a Gorenstein local ring, let $M$ be a finitely generated $A$-module of dimension $d$.

1. Assume that $d \geq 3$ and $\operatorname{depth}(M)>0$, then $\operatorname{depth} D^{d-1}(M)=0$ if and only if $\operatorname{depth} K_{M}=2$.

2. Assume that $d \geq 2$, depth $(M)=d-1$, and $D^{d-1}(M)$ has dimension $d-2$. Then $\operatorname{depth} D^{d-1}(M)=$ depth $K_{M}-2$.

In particular suppose that $\operatorname{depth}(M)=d-1$, and $\operatorname{dim} D^{d-1}(M)=d-2$. Then $D^{d-1}(M)$ is a Cohen-Macaulay module if and only if the canonical module $K_{M}$ is Cohen-Macaulay.

Let $a \in m$ be a non zero divisor of $M$. From the exact sequence :

$$
0 \longrightarrow M \stackrel{\times a}{\longrightarrow} M \longrightarrow M / a M \longrightarrow 0
$$

we get the following long exact sequence:

$0 \rightarrow D^{d}(M) \stackrel{\times a}{\rightarrow} D^{d}(M) \stackrel{\alpha}{\rightarrow} D^{d-1}(M / a M) \stackrel{\beta}{\rightarrow} D^{d-1}(M) \stackrel{\times a}{\rightarrow} D^{d-1}(M) \rightarrow D^{d-2}(M / a M) \rightarrow D^{d-2}(M) \rightarrow \ldots$

From this exact sequence we get the short exact sequences:

$$
\begin{gathered}
0 \longrightarrow D^{d}(M) \stackrel{\times a}{\longrightarrow} D^{d}(M) \longrightarrow \operatorname{Im} \alpha \longrightarrow 0 \\
0 \longrightarrow \operatorname{Im} \alpha \longrightarrow D^{d-1}(M / a M) \longrightarrow \operatorname{Im} \beta \longrightarrow 0
\end{gathered}
$$

Note that $\operatorname{Im} \beta=\left(0:_{D^{d-1}(M)} a\right)$. From the exact sequence 2 we have the long local cohomology sequence:

$$
0 \longrightarrow H_{\mathrm{m}}^{0}(\operatorname{Im} \alpha) \longrightarrow H_{\mathrm{m}}^{0}\left(D^{d-1}(M / a M)\right) \longrightarrow H_{\mathrm{m}}^{0}\left(\left(0:_{D^{d-1}(M)} a\right)\right) \longrightarrow H_{\mathrm{m}}^{1}(\operatorname{Im} \alpha) \longrightarrow 0,
$$

where $H_{\mathrm{m}}^{0}\left(D^{d-1}(M / a M)\right)=H_{\mathrm{m}}^{1}\left(D^{d-1}(M / a M)\right)=0$, since $\operatorname{dim} M / a M=d-1 \geq 2$ and $D^{d-1}(M / a M)$ satisfies condition $S_{2}$, hence the map $H_{\mathrm{m}}^{0}\left(\left(0:_{D^{d-1}}(M) a\right)\right) \longrightarrow H_{\mathrm{m}}^{1}(\operatorname{Im} \alpha)$ is an isomorphism.

1. If depth $K_{M}=2$, suppose first that depth $D^{d-1}(M)>0$, then we can choose $a \in m$, a non zero divisor for depth $D^{d-1}(M)$, this will imply that $H_{\mathrm{m}}^{1}(\operatorname{Im} \alpha)=0$ and then depth $K_{M} \geq 3$. A contradiction.

If depth $D^{d-1}(M)=0$ we have either $\operatorname{dim} D^{d-1}(M)=0$ or not. If $\operatorname{dim} D^{d-1}(M)=0$ then the module $\left(0:_{D^{d-1}(M)} a\right)$ is non null but has also dimension zero. If $\operatorname{dim} D^{d-1}(M)>0$ then

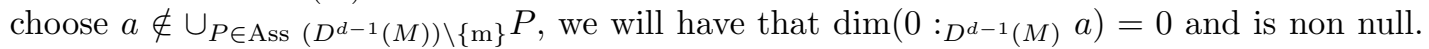
In both cases $H_{\mathrm{m}}^{1}(\operatorname{Im} \alpha) \simeq H_{\mathrm{m}}^{0}\left(\left(0:_{D^{d-1}(M)} a\right)\right) \neq 0$ and so depth $K_{M}=2$.

2. We will prove the claim by induction on $d$. Remark that if $\operatorname{dim} M=2$, our statement is true. In fact following Section 1, the canonical module is Cohen-Macaulay of dimension two and since by our hypothesis $D^{1}(M)$ is of dimension 0 , it is Cohen Macaulay. Let $d \geq 3$, by the first claim we can assume that depth $D^{d-1}(M)>0$.

Let $a \in m$ be a non zero divisor for both $M$ and $D^{d-1}(M)$. Since $a$ is a non zero divisor for $D^{d-1}(M)$, we have $\beta=0$ and we get two exact sequences:

$$
0 \longrightarrow D^{d}(M) \stackrel{\times a}{\longrightarrow} D^{d}(M) \stackrel{\alpha}{\longrightarrow} D^{d-1}(M / a M) \longrightarrow 0
$$




$$
0 \longrightarrow D^{d-1}(M) \stackrel{\times a}{\longrightarrow} D^{d-1}(M) \longrightarrow D^{d-2}(M / a M) \longrightarrow 0
$$

It then follows that $M / a M$ satisfies the induction hypothesis. Hence depth $D^{d-1}(M / a M)=$ depth $D^{d-2}(M / a M)+2$, the above two short exact sequences imply then that depth $D^{d}(M)=$ depth $D^{d-1}(M)+2$.

This ends the proof of the theorem. As a consequence of the proof we have:

Corollary 1 Let $(A, \mathrm{~m})$ be a factor ring of a Gorenstein local ring, let $M$ be a finitely generated $A$-module with $\operatorname{dim} M=d \geq 3$ and $\operatorname{depth}(M)>0$. If $\operatorname{dim} D^{d-1}(M)>0$, let $a \in m$ be a non zero divisor of $M$ and $a \notin \cup_{P \in \operatorname{Ass}}\left(D^{d-1}(M)\right) \backslash\{\mathrm{m}\} P$, then $K_{M} / a K_{M}$ is isomorphic to $K_{M / a M}$ if and only if depth $K_{M} \geq 3$. In particular if $K_{M}$ is a Cohen-Macaulay module then $K_{M / a M}$ is a Cohen-Macaulay module.

Proof .- With the above notations, $K_{M} / a K_{M}$ is isomorphic to $K_{M / a M}$ if and only if $\operatorname{Im} \beta=$ $\left(0:_{D^{d-1}(M)} a\right)=0$. By our choice of $a$, we have that $\operatorname{dim}\left(0:_{D^{d-1}(M)} a\right)=0$, so $H_{\mathrm{m}}^{0}\left(\left(0:_{D^{d-1}(M)} a\right)\right)=$ $\left(0:_{D^{d-1}(M)} a\right)$ and then $\operatorname{Im} \beta=0$ if and only if $H_{\mathrm{m}}^{1}(\operatorname{Im} \alpha)=0$, this is equivalent to depth $K_{M} \geq 3$.

Example 1 (See also[10]) Consider the semigroup in $S \subset \mathbb{N}^{3}$ generated by the elements $(3,0,0),(2,1,0)$, $(0,3,0),(3,0,1),(2,1,1),(0,3,1)$, the semigroup ring $K[S]$ has dimension 3, codimension 3 , and depth $K[S]=2$, the ring $K[S]$ satisfies the condition $S_{2}$ of Serre so it is isomorphic to $D^{3}\left(D^{3}(K[S])\right)$. The canonical module $D^{3}(K[S])$ is not Cohen-Macaulay. Remark that we have $\operatorname{dim} D^{2}(K[S])=0$.

\section{$3 \quad S_{2}$-fication of unmixed modules}

Let $(A, \mathrm{~m})$ be a noetherian local ring, (resp. graded), quotient of a Gorenstein local ring (resp. graded Gorenstein ring) and $M$ be an $A$-module of dimension $d$.

We recall that if $M$ is unmixed, the module $D^{d}\left(D^{d}(M)\right)$ satisfies the condition $S_{2}$ and we have an exact sequence :

$$
0 \longrightarrow M \longrightarrow D^{d}\left(D^{d}(M)\right) \longrightarrow M^{\prime \prime} \longrightarrow 0
$$

with $\operatorname{dim} M^{\prime \prime} \leq d-2$. Moreover if there exist an $A$-module $M^{\prime}$ of dimension $d$, satisfying the condition $S_{2}$ and an exact sequence :

$$
0 \longrightarrow M \longrightarrow M^{\prime} \longrightarrow M^{\prime} / M \longrightarrow 0
$$

with $\operatorname{dim} M^{\prime} / M \leq d-2$, then $M^{\prime} \simeq D^{d}\left(D^{d}(M)\right)$. The $A$-module $M^{\prime}$ is the $S_{2}$-fication of $M$ and if $M^{\prime}$ is a Cohen-Macaulay module it is a Macaulayfication of $M$.

Lemma 1 Set $M^{\prime}:=D^{d}\left(D^{d}(M)\right)$. Assume that $M$ is unmixed not satisfying the condition $S_{2}$, then:

A) The canonical module $K_{M}=D^{d}(M)$ is a Cohen-Macaulay module if and only if $M^{\prime}$ it is.

B) If $K_{M}$ is a Cohen-Macaulay module, then:

$H_{\mathrm{m}}^{i-1}\left(M^{\prime} / M\right) \simeq H_{\mathrm{m}}^{i}(M)$ for $i=1, \ldots, d-1$. In particular $\operatorname{depth}\left(M^{\prime} / M\right)=\operatorname{depth} M-1$ and $\operatorname{dim} M^{\prime} / M=\max \left\{i \leq d-2 / H_{\mathrm{m}}^{i+1}(M) \neq 0\right\}$

As a special case $M^{\prime} / M$ is a Cohen-Macaulay Module if and only if only one of the local cohomology modules $H_{\mathrm{m}}^{i}(M), i \leq n-1$, does not vanish.

In this case the Matlis dual $D^{i}(M)$ of $H_{m}^{i}(M)$ is a Cohen Macaulay module of dimension $i-1$. In particular if depth $M=d-1$, then $M^{\prime} / M$ is a Cohen-Macaulay Module of dimension $d-2$, and $D^{d-1}(M)$ is a Cohen-Macaulay module of dimension $d-2$. 
C) The Non-Cohen-Macaulay locus of $M$ is given by $\operatorname{Supp}\left(M^{\prime} / M\right)$.

\section{Proof .-}

A) Since $\operatorname{dim} M^{\prime} / M \leq n-2$ we have $D^{d}(M) \simeq D^{d}\left(M^{\prime}\right)$, if $M^{\prime}$ is Cohen-Macaulay, then $D^{d}\left(M^{\prime}\right)$ is a Cohen-Macaulay module, hence the canonical module $K_{M}$ is Cohen-Macaulay of dimension $d$. The converse follows since $M^{\prime} \simeq D^{d}\left(D^{d}\left(M^{\prime}\right)\right)$

B) From the long exact sequence of the local cohomology associated to the sequence:

$$
0 \longrightarrow M \longrightarrow M^{\prime} \longrightarrow M^{\prime} / M \longrightarrow 0
$$

we get $H_{\mathrm{m}}^{i-1}\left(M^{\prime} / M\right) \simeq H_{\mathrm{m}}^{i}(M)$ for $i=1, \ldots, d-1$, which implies B).

C) The above exact sequence is still exact by localization on any prime ideal $P$; on the other hand $K(K(M))_{P}=K\left(K\left(M_{P}\right)\right)$ and recall that if $M$ is Cohen-Macaulay then the natural map $M \longrightarrow K(K(M))$ is an isomorphism. It follows that the Non-Cohen-Macaulay locus of $M$ is given by Supp $\left(M^{\prime} / M\right)$.

When $M$ is unmixed we get a new version of theorem 2

Theorem 3 Let $M$ be unmixed of dimension d, not satisfying the condition $S_{2}$, and depth $M=$ $d-1$, then $\operatorname{dim} D^{d-1}(M)=d-2$, and depth $D^{d-1}(M)=\operatorname{depth} K_{M}-2$. In particular $D^{d-1}(M)$ is Cohen-Macaulay if and only if $K_{M}$ is Cohen-Macaulay.

Proof .- In regard of Theorem 2, we need only to prove that $\operatorname{dim} D^{d-1}(M)=d-2$.

Set $M^{\prime}=D^{d}\left(D^{d}(M)\right)$, from the exact sequence :

$$
0 \longrightarrow M \longrightarrow M^{\prime} \longrightarrow M^{\prime} / M \longrightarrow 0
$$

with $\operatorname{dim} M^{\prime} / M \leq d-2$, we have $D^{d}(M) \simeq D^{d}\left(M^{\prime}\right)$. Since $M^{\prime}$ satisfies $S_{2}$ we have $\operatorname{dim} D^{d-j}\left(M^{\prime}\right) \leq$ $d-j-2$, for all $0 \leq j \leq d-1$.

Assume that $\operatorname{dim} M^{\prime} / M=d-k<d-2$, for some $3 \leq k \leq d-1$, then from the long exact sequence associated to the above short exact sequence we have:

$$
0 \longrightarrow D^{d-k}\left(M^{\prime} / M\right) \longrightarrow D^{d-k}\left(M^{\prime}\right) \longrightarrow 0
$$

this carries a contradiction since $d-k=\operatorname{dim} M^{\prime} / M=\operatorname{dim} D^{d-k}\left(M^{\prime}\right)=d-k \leq d-k-2$.

From the exact sequence

$$
0 \longrightarrow D^{d-1}\left(M^{\prime}\right) \longrightarrow D^{d-1}(M) \longrightarrow D^{d-2}\left(M^{\prime} / M\right) \longrightarrow D^{d-2}\left(M^{\prime}\right) \longrightarrow 0
$$

we get $\operatorname{dim} D^{d-1}(M)=\operatorname{dim} D^{d-2}\left(M^{\prime} / M\right)=d-2$ since $\operatorname{dim} D^{d-j}\left(M^{\prime}\right) \leq d-j-2$, for all $0 \leq j \leq$ $d-1$.

Remark 1 Let $M$ be a finitely generated graded module over a ring of polynomials, with $\operatorname{dim} M=d$, and depth $M=d-1$. It is well known that if $0 \longrightarrow G \stackrel{\phi}{\longrightarrow} F \longrightarrow \ldots$ is the last term of the minimal syzygies of $M$ then $\ldots \longrightarrow F \stackrel{\sigma}{\longrightarrow} G \longrightarrow D^{d-1}(M) \longrightarrow 0$ is a presentation of $D^{d-1}(M)$, where $\sigma$ is the matrix transpose of $\phi$. 
Example 2 Let $A$ be the affine ring of the projective surface in $P^{4}$ defined parametrically by:

$$
a=s^{4}+t^{4}, b=s^{2} t u, c=s^{3} t, d=s t^{3}, e=s u^{3}
$$

then depth $A=2$ and $\sigma=\left(a, c^{2}+d^{2}, c e, b^{3}\right)$. It follows that $D^{2}(A)$ is Cohen-Macaulay of dimension 1 , and the $S_{2}-$ fication is in fact a Macaulayfication. It is not difficult to check that

$$
A^{\prime}=K\left[s^{4}+t^{4}, s^{2} t u, s^{3} t, s t^{3}, s u^{3}, s^{2} t^{2}\right]
$$

is the Macaulayfication of $A$.

Example 3 Let $A$ be the affine ring of the projective surface in $P^{4}$ defined parametrically by:

$$
a=s^{4}, b=s^{3} t+u^{4}, c=s^{2} t^{2}, d=s u^{3}, e=t^{2} u^{2}
$$

a quick computation with Macaulay, if $\operatorname{char}(K) \neq 2,3$, gives that depth $A=2$ and $\sigma$ is given by

$$
\left(\begin{array}{ccccccc}
0 & -a e & d^{2} & -c & b & 0 & 0 \\
2 a b^{3}-2 a^{2} b c+d^{4} & 3 / 2 a b^{3}+1 / 2 a^{2} b c & a^{2} b^{2}-a^{3} c & -3 / 2 b d^{2}-a^{2} e & -1 / 2 a d^{2} & e & 6 c
\end{array}\right),
$$

in this case $\mathrm{m}$ is an associated prime ideal of the ideal generated by the entries of the second row of $\sigma$, but again using Macaulay we get that that $D^{2}(A)$ is Cohen-Macaulay of dimension 1. Also in this example the $S_{2}$-fication is in fact a Macaulayfication. We can check that

$$
A^{\prime}=K\left[s^{4}, s^{3} t+u^{4}, s^{2} t^{2}, s u^{3}, t^{2} u^{2}, s^{2} u^{2}\right]
$$

is the Macaulayfication of $A$.

\section{Lattice and toric ideals}

Let $R=K\left[x_{1}, \ldots, x_{m}\right]$ be a polynomial ring, $L \subset \mathbb{Z}^{m}$ a lattice of rank $r$. We assume that $L$ is a positive lattice, that is, every non zero vector in $L$ has positive and negative coordinates. We can write every vector $\mathbf{u}$ in $\mathbb{Z}^{m}$ uniquely as $\mathbf{u}=\mathbf{u}_{+}-\mathbf{u}_{-}$, where $\mathbf{u}_{+}$and $\mathbf{u}_{-}$are non-negative and have disjoint support. Set $I_{L} \subset R$ be the ideal generated by all the binomials $x^{\mathbf{u}_{+}}-x^{\mathbf{u}_{-}}$, where $\mathbf{u}$ runs over all vectors of $L . I_{L} \subset R$ is called a lattice ideal associated to $L$. Let Sat $L=\left\{\mathbf{u} \in \mathbb{Z}^{m} \mid\right.$ $k \mathbf{u} \in L$ for some $k \in \mathbb{Z}\}$. The group $\mathbb{Z}^{m} / L$ is a finitely generated abelian group, and $I_{L} \subset R$ is a prime ideal if and only if $\mathbb{Z}^{m} / L$ has no torsion. We quote the following theorem from the proof of Corollaries 2.2 and 2.5 of $[3]$ :

Theorem 4 Let $K$ be an algebraically closed field of any characteristic $p \geq 0$. The ideal $I_{L} \subset R$ is always unmixed. Moreover any $x_{i}$ is a non zero divisor modulo $I_{L}$.

When the ideal $I_{L} \subset R$ is prime it is called toric. In the toric case the lattice $L$ is usually viewed as the lattice of the relations of a finitely generated semigroup $S \subset \mathbb{N}^{n}$. In general we have an isomorphism $\mathbb{Z}^{m} / L \longrightarrow \mathbb{Z}^{d} \oplus H$, where $H$ is a finite group, the images $\mathbf{a}_{\mathbf{1}} \ldots, \mathbf{a}_{\mathbf{m}}$ of the canonical basis of $\mathbb{Z}^{m}$ under this isomorphism generate a finitely generated semigroup $S \subset \mathbb{Z}^{n} \oplus H$, which generates $G(S):=\mathbb{Z}^{d} \oplus H$. In fact $K[S]:=R / I_{L}=\oplus_{g \in S} K \underline{t}^{g_{1}} \underline{u}^{g_{2}}$, where $g_{1} \in \mathbb{Z}^{n}, g_{2} \in H$ and $g=\left(g_{1}, g_{2}\right)$.

We set $\tilde{S}$ the projection of $S$ in $\mathbb{Z}^{d}$, let $\mathcal{C}_{S}$ be the cone generated by $\tilde{S}$ in $Q^{d}$, and $F_{1}, F_{2}, \ldots, F_{l}$ its faces of dimension $d-1$. Let $S_{i}=\left\{x-y ; x, y \in S, \tilde{y} \in \tilde{S} \cap F_{i}\right\} \subset \mathbb{Z}^{n} \oplus H$. 
Let $L$ be any lattice, corresponding to the semigroup $S \subset G(S)$, as in [5], we will define another semigroup $S^{\prime}=\cap S_{i} \subset G(S)$ such that the semigroup ring $K\left[S^{\prime}\right]$ is the $S_{2}$-fication of the semigroup $\operatorname{ring} K[S]:=R / I_{L}$. Moreover if $S$ is simplicial then $K\left[S^{\prime}\right]$ is the Macaulayfication of the semigroup ring $K[S]:=R / I_{L}$. This extends to the lattice case a theorem of [5]. First we extends some preliminary results from [10, to the lattice case, the proofs are very similar and we let it to the reader.

1. Let $\bar{S}=\left\{z \in G(S), \exists p \in \mathbb{N}^{*}, p z \in S\right\}$, then $K[\bar{S}]$ is the normalization of $K[S]$.

2. A semigroup $S \subset \mathbb{Z}^{l} \oplus H$, is called standard if the following conditions are satisfied:

(a) $\bar{S}=G(S) \cap \mathbb{N}^{l} \oplus H$,

(b) $S_{(i)} \neq S_{(j)}$ for $i \neq j$, where $S_{(i)}=\left\{x \in S ; x_{i}=0\right\}$ and $x=\left(x_{1}, \ldots, x_{l}, h\right)$, with $h \in H$.

(c) $\operatorname{rank}_{\mathbb{Z}} G\left(S_{(i)}\right)=\operatorname{rank}_{\mathbb{Z}} G(S)-1, i=1, \ldots, l$.

By the Hochster's transformation, see [10, there is an standard semigroup $T(S)$ isomorphic to $S$, also by this transformation $T\left(S^{\prime}\right)=T(S)^{\prime}$. So we can assume that our semigroup $S$ is standard.

3. The polynomial ring $R$ has two gradings, it is $G(S)=\mathbb{Z}^{m} / L=\mathbb{Z}^{d} \oplus H$-graded: two monomials $\underline{x}^{\alpha}, \underline{x}^{\beta}$ have the same grading if and only if the vector $\alpha-\beta \in L$, the lattice ideal $I_{L}$ is $\mathbb{Z}^{d} \oplus H$-graded. The polynomial ring $R$ is $\mathbb{Z}^{d}$-graded by grouping all homogeneous elements with the same $\mathbb{Z}^{d}$-graded component.

Example 4 The minimal primes ideals of $I_{L}$ are $\mathbb{Z}^{d}$-graded, but not necessarily $\mathbb{Z}^{d} \oplus H-$ graded. Let $I=\left(x^{2}-y^{2}\right) \subset K[x, y]$, in this case $L=\mathbb{Z}(2,-2)$ and the isomorphism $\mathbb{Z}^{2} / L \longrightarrow$ $\mathbb{Z} \oplus \mathbb{Z} / 2 \mathbb{Z}$, is given by $(a, b) \mapsto(a+b, b \bmod 2)$; it follows that $\operatorname{deg}(x)=(1,0), \operatorname{deg}(y)=(1, \overline{1})$. The minimal primary decomposition of $I$ is given by $\left(x^{2}-y^{2}\right)=(x-y)(x+y)$.

Let $A$ be any arbitrary subset of $G(S)$, we will denote by $K[A]$ the $K$-vector space spanned by $A$ in $K[G(S)]$. If $A+S \subset A$, we will call $A$ an $S$-ideal. A proper subset $P$ of $S$ is a prime ideal if $P$ is an $S$-ideal and $S \backslash P$ is additively closed. Every $G(S)$-graded prime ideal $\underline{p}$ of $K[S]$ is exactly of the form $K[P]$ for some prime ideal $P$ of $S$ and the homogeneous localization $K[S]_{\underline{p}}$ is isomorphic to $K[S-(S \backslash P)]$.

4. Let $S \subset \mathbb{Z}^{l} \oplus H$ be a standard semigroup with torsion, and let $I$ be a nonempty subset of $[1, l]$, set $P_{I}=\left\{x \in S ; x_{i}>0\right.$ for some $\left.i \in I\right\}$ and $p_{I}=K\left[P_{I}\right]$. Then the set $\left\{P_{I}\right\}$ is the set of prime ideals of $S$ (see the proof of the next lemma). Moreover $K[S] / p_{\{i\}}=K\left[S_{(i)}\right]$ and if $J \subset I$ then $P_{J} \subset P_{I}$. This implies that $p_{\{1\}}, \ldots, p_{\{l\}}$ are the unique $G(S)$-graded prime ideals of height one of $K[S]$.

The following Lemma shows that the extension from the toric case to the lattice case is non trivial:

Lemma 2 Let $\mathcal{P} \subset K[S]$ be an $\mathbb{Z}^{d}$-graded prime ideal of height $>0$. Then $\mathcal{P}$ is $G(S)$-graded and $\mathcal{P}=p_{I}$ for some non empty subset $I$.

Proof .- First, let remark that if $z_{1}, z_{2} \in K[S]$ are two pure monomials with the same $\mathbb{Z}^{d}$-grade, then $z_{1}^{h}-z_{2}^{h}=0$, where $h$ is the order of the group $H$, and this imply that for any prime ideal $\mathbf{p}$ in $K[S]$ there exists $\xi$ a $h$-root of unity such that $z_{1}+\xi z_{2} \in \mathbf{p}$ 
We prove that $\mathcal{P}$ contains one monomial element $t^{g_{1}} \underline{u}^{g_{2}}$ for some $\left(g_{1}, g_{2}\right) \in S$.

Since $\operatorname{ht}(\mathcal{P}) \geq 1$, and because $I_{L}$ is unmixed, $\mathcal{P}$ contains $Q$ an associated prime of $I_{L}$ and a non zero divisor $z$ for $K[S]$. Now let $z \in \mathcal{P}$ be a non zero divisor, we can assume that $z$ is $\mathbb{Z}^{n}$-homogeneous, if $z$ is not monomial we can write it as a sum of monomials $z=\lambda_{1} z_{1}+\ldots+\lambda_{r} z_{r}$, with coefficients $\lambda_{i} \in K$, then for any $i=1, \ldots, r$ there exists $h$-roots of unity $\xi_{i}$, such that $z_{i}+\xi_{i} z_{1} \in Q \subset \mathcal{P}$, but

$$
z=\sum_{i=1}^{r} \lambda_{i}\left(z_{i}+\xi_{i} z_{1}\right)-\left(\sum_{i=1}^{r} \lambda_{i} \xi_{i}\right) z_{1},
$$

and since $z \notin Q$ we have $\sum_{i=1}^{r} \lambda_{i} \xi_{i} \neq 0$, which implies that $z_{1} \in \mathcal{P}$, and we are done.

The same proof shows that for any non empty set $I$, if $\mathcal{P} \not \subset p_{I}$, then we can choose a monomial element $z \in \mathcal{P} \backslash p_{I}$.

Let $I$ be the set of integers $i \in[1, \ldots, l]$ such that $p_{\{i\}}$ is contained in $\mathcal{P}$, we will prove that $I$ is non empty and $p_{I}=\mathcal{P}$.

It is clear that $p_{I} \subset \mathcal{P}$, remark that if $I=[1, \ldots, l]$, then $\mathcal{P}$ contains the unique graded maximal ideal of $K[S]$. So we can assume that $I$ is a proper subset of $[1, \ldots, l]$. Suppose a contrario that there exist $z \in \mathcal{P} \backslash p_{I}$, (If $I$ is empty choose $z$ any monomial non zero divisor), we can assume that $z$ is pure monomial, and if $z=\underline{t}^{a} \underline{u}^{b}$, then $a_{i}=0$ for all $i \in I$, for any $j \notin I$ choose a monomial $\underline{t}^{c^{j}} \underline{u}^{d^{j}} \in p_{\{j\}} \backslash \mathcal{P}$, let $c=\sum_{j \notin I} c^{j}, d=\sum_{j \notin I} d^{j}$, then $c_{j}>0$ for any $j \notin I$, and there exist a positive integer $p$ such that $p(c, d)-(a, b) \in \mathbb{N}^{l} \oplus H \cap G=\bar{S}$, and for some positive integer $k$, $k p(c, d)-k(a, b) \in S$. It follows then that $\prod_{i \notin I}\left(\underline{t}^{c^{j}} \underline{u}^{d^{j}}\right)^{p k} \in \mathcal{P}$ and $\underline{t}^{c^{j}} \underline{u}^{d^{j}} \in \mathcal{P}$, for some $j$. A contradiction.

Theorem 5 Assume that the semigroup (eventually with torsion) $S$ is standard. Let $G(S)$ be the group generated by $S$ of rank d, let $S^{\prime}:=\cap_{i=1}^{l}\left(S-\left(S \backslash P_{i}\right)\right)$ be a subsemigroup of $G(S)$, where $S \backslash P_{i}$ consist of the elements in $S$, which the $i$ coordinate is 0 . Then

$$
K\left[S^{\prime}\right]=\cap_{i=1}^{l} K[S]_{\left(p_{\{i\}}\right)}
$$

and $K\left[S^{\prime}\right]$ satisfies the condition $S_{2}$. Let remark that $K[S]_{\left(p_{\{i\}}\right)}$ is a homogeneous localization and the intersection is taken in the localization $T^{-1} K[S]$, where $T$ is the set of all pure monomials, also since $I_{L}$ is a lattice ideal any monomial is a non zero divisor for $K[S]$.

We also have an exact sequence :

$$
0 \longrightarrow K[S] \longrightarrow K\left[S^{\prime}\right] \longrightarrow K\left[S^{\prime} \backslash S\right] \longrightarrow 0
$$

and $\operatorname{dim} K\left[S^{\prime} \backslash S\right] \leq d-2$. Moreover if $S$ is simplicial then $K\left[S^{\prime}\right]$ is a Cohen-Macaulay ring.

Proof .- It follows from [5], p.244, that the property $S_{k}$ holds for a $\mathbb{Z}^{d}$ graded module $M$ if and only if

$$
\operatorname{depth} M_{(p)} \geq \min \left\{k, \operatorname{dim} M_{(p)}\right\}
$$

for any $\mathbb{Z}^{d}$ homogeneous prime ideal $p$.

As a consequence the ring $\cap_{h t(p)=1} K[S]_{(p)}$, where $p$ runs over all $\mathbb{Z}^{d}$ homogeneous prime ideals in $K[S]$ of height one, satisfies the condition $S_{2}$. Now the above lemma proves that $\left\{p_{\{1\}}, \ldots, p_{\{l\}}\right\}$ are all the $\mathbb{Z}^{d}$ homogeneous prime ideals in $K[S]$ of height one and then

$$
K\left[S^{\prime}\right]=\cap_{i=1}^{l} K[S]_{\left(p_{\{i\}}\right)},
$$


satisfies the condition $S_{2}$. Also we have that $K[S]_{\left(p_{\{i\}}\right)}=K\left[S^{\prime}\right]_{\left(p_{\{i\}}\right)}$ and since the module $K\left[S^{\prime} \backslash S\right]$ is $\mathbb{Z}^{d}$-graded we get $\operatorname{dim} K\left[S^{\prime} \backslash S\right] \leq d-2$.

If $S$ is simplicial, let $x_{1}, \ldots, x_{d}$ be the variables in $R$ corresponding to the extreme rays of $\mathcal{C}_{S}$, then $S^{\prime}$ is also simplicial and $x_{1}, \ldots, x_{d}$ are parameters for both $K[S], K\left[S^{\prime}\right]$, since $S^{\prime}$ satisfies the condition $S_{2}$ we have that any pair $x_{i}, x_{j}$ is a regular sequence in $K\left[S^{\prime}\right]$, if we have a relation $f x_{i}=\sum_{j<i} f_{j} x_{j}$ then because of the grading we certainly have $f x_{i}=f_{j} x_{j}$ for some $j$, this implies that the sequence $x_{1}, \ldots, x_{d}$ is a regular sequence in $K\left[S^{\prime}\right]$, so $K\left[S^{\prime}\right]$ is a Cohen-Macaulay ring.

As a Corollary we have

Corollary 2 Let $L \subset \mathbb{N}^{m}$ be a positive lattice of rank $r$, set $\operatorname{dim} R / I_{L}=d=m-r$. If $\operatorname{depth} R / I_{L}=$ $d-1$ then $\operatorname{dim} K\left[S^{\prime}-S\right]=d-2$, depth $D^{d-1}(K[S])=\operatorname{depth} K_{K[S]}-2$, and the following are equivalent:

1. $D^{d}\left(D^{d}(K[S])\right)$ is Cohen-Macaulay.

2. the canonical module of $K[S]$ is Cohen-Macaulay.

3. the module $D^{d-1}(K[S])$ is Cohen-Macaulay.

The proof is immediate from Theorem 2 .

Corollary 3 Let $S \subset \mathbb{N}^{n} \oplus H$ be a simplicial finitely generated semigroup of rank $d$, then

1. $D^{d}\left(D^{d}(K[S])\right)$ is Cohen-Macaulay

2. the canonical module of $K[S]$ is Cohen-Macaulay

We review the following example from [7], Example B.1:

Example 5 Let $K$ be a field, $A$ the affine semigroup ring

$$
K\left[a, b, c, d, e^{2}, e^{3}, a d e, b d e, c d e, d^{2} e\right] .
$$

We can see immediately that $K\left[S^{\prime}\right]=K[a, b, c, d, e]$ and then it is a Macaulayfication of $A$, and we get the following exact sequence (see also [7]),

$$
0 \longrightarrow A \longrightarrow K[a, b, c, d, e] \longrightarrow C[-1] \longrightarrow 0,
$$

where $C=A /\left(a d, b d, c d, d^{2}, e^{2}, e^{3}, a d e, b d e, c d e, d^{2} e\right)$ has dimension three. It follows that the Non Cohen-Macaulay locus of $A$ is the support of $C$.

Example 6 The following example is a toric ring of codimension two and dimension 4, which canonical module is not Cohen-Macaulay. The ideal $I_{L} \subset K[a, b, c, d, e, f]=R$ has the following generators:

$$
\begin{gathered}
a b^{4} c-d e^{3} f^{2}, b c^{3} d^{3}-a^{2} e^{2} f^{3}, c^{2} d^{4} e-a^{3} b^{3} f, b^{5} c^{4} d^{2}-a e^{5} f^{5}, \\
a^{4} b^{7}-c d^{5} e^{4} f, c^{5} d^{7}-a^{5} b^{2} e f^{4}, b^{9} c^{5} d-e^{8} f^{7} .
\end{gathered}
$$

Let $0 \longrightarrow G \stackrel{\phi}{\longrightarrow} F$ be the last term of a resolution of $A:=S / I_{L}, \sigma$ be the transpose of $\phi$, then $F \stackrel{\sigma}{\longrightarrow} G \longrightarrow D^{3}(A) \longrightarrow 0$ is a presentation of module $D^{3}(A)$, a quick computation by Macaulay gives that 


$$
\sigma=\left(\begin{array}{cccccccccc}
e^{2} f^{2} & -b c & 0 & d & -a & 0 & 0 & 0 & 0 & 0 \\
a b^{3} & -d e & -f & 0 & 0 & c & 0 & 0 & 0 & 0 \\
c^{2} d^{3} & -a^{2} f & 0 & 0 & 0 & 0 & e & -b & 0 & 0 \\
0 & 0 & 0 & b^{4} c & -e^{3} f^{2} & 0 & 0 & 0 & -d & a
\end{array}\right)
$$

and that the module $D^{3}(A)$ has dimension 2, but depth $D^{3}(A)=1$. So the canonical module of $S / I_{L}$ is not Cohen-Macaulay, in fact depth $K_{A}=3$.

In what follows we will write $I$ instead $I_{L}$.

Theorem 6 Let $R=K\left[x_{1}, \ldots, x_{n}\right]$ be a polynomial ring, let $A=R / I$ be a lattice ring, of codimension two and dimension $d$. If $I$ is minimally generated by 4 generators, then $D^{d-1}(A)$ is a complete intersection. In particular, the canonical ring $K_{A}$ is Cohen-Macaulay of dimension $d$, and the $S_{2}-$ fication is a Macaulayfication of $A$. The Non-Cohen-Macaulay locus of $A$ is the support of a Cohen-Macaulay module of dimension $d-2$.

Proof .- The resolution of $A$, follows from 9] Construction 5.2:

$$
0 \longrightarrow R \stackrel{\phi}{\longrightarrow} R^{4} \longrightarrow R^{4} \longrightarrow R \longrightarrow A \longrightarrow 0
$$

where $\sigma$ the transpose of $\phi$ is given by:

$$
\sigma=\left(\begin{array}{llll}
-x^{\mathbf{s}} & x^{\mathbf{t}} & x^{\mathbf{r}} & -x^{\mathbf{p}}
\end{array}\right)
$$

where all monomials have disjoints supports. Then the entries of $\sigma$ define a complete intersection, that is $D^{d-1}(A)$ is a complete intersection. The rest of the proof follows from Lemma 2

Question Let $R=K\left[x_{1}, \ldots, x_{d+2}\right]$ be a polynomial ring, let $A=R / I$ be a lattice ring of codimension two and dimension $d$, is it true that $D^{d-1}(A)$ has non zero divisors?

\section{$5 \quad$ Simplicial lattices ideals of height 2}

Let $K$ be a field and $R:=K\left[y, z, x_{1}, \ldots, x_{n}\right]$ the ring of polynomials in the variables $y, z, x_{1}, \ldots, x_{n}$. Let $a_{i}, b_{i}, c_{i} 1 \leq i \leq n$ be naturals numbers satisfying the conditions:

$$
a_{i} \neq 0,\left(b_{i}, c_{i}\right) \neq 0 \forall i,\left(b_{1}, \ldots, b_{n}\right) \neq 0,\left(c_{1}, \ldots, c_{n}\right) \neq 0
$$

For $i=1, \ldots, n$ let $\mathbf{d}_{\mathbf{i}}=a_{i} \mathbf{e}_{\mathbf{i}}$, where $\mathbf{e}_{\mathbf{n}}, \ldots, \mathbf{e}_{\mathbf{n}}$ is the canonical basis of $\mathbb{N}^{n}$, and $\mathbf{a}_{\mathbf{1}}=$ $\left(b_{1}, \ldots, b_{n}\right), \mathbf{a}_{2}=\left(c_{1}, \ldots, c_{n}\right)$. Let $H$ be a finite abelian group and $h_{1}, \ldots, h_{n+2} \in H$ that generates it. Let $S$ be the subsemigroup of $\mathbb{N}^{n} \oplus H$ generated by

$$
\left(\mathbf{d}_{\mathbf{1}}, \mathbf{h}_{\mathbf{1}}\right), \ldots,\left(\mathbf{d}_{\mathbf{n}}, \mathbf{h}_{\mathbf{n}}\right),\left(\mathbf{a}_{\mathbf{1}}, \mathbf{h}_{\mathbf{n}+\mathbf{1}}\right),\left(\mathbf{a}_{\mathbf{2}}, \mathbf{h}_{\mathbf{n}+\mathbf{2}}\right) .
$$

Definition 1 A simplicial lattice ideal of height two is the lattice ideal $I_{L} \subset R$, where:

$$
L=\left\{\mathbf{w} \in \mathbb{Z}^{n+2}, w_{1}\left(\mathbf{d}_{\mathbf{1}}, \mathbf{h}_{\mathbf{1}}\right)+\ldots+w_{n}\left(\mathbf{d}_{\mathbf{n}}, \mathbf{h}_{\mathbf{n}}\right)+w_{n+1}\left(\mathbf{a}_{\mathbf{1}}, \mathbf{h}_{\mathbf{n}+\mathbf{1}}\right)+w_{n+2}\left(\mathbf{a}_{\mathbf{2}}, \mathbf{h}_{\mathbf{n}+\mathbf{2}}\right)=0\right\} .
$$

We remark that the last two coordinates of vectors in $L$, determine all the lattice $L$. More precisely, consider the group morphism:

$$
\Phi: \mathbb{Z}^{2} \longrightarrow \mathbb{Z} / a_{1} \mathbb{Z} \times \ldots \times \mathbb{Z} / a_{n} \mathbb{Z} \quad(s, p) \mapsto\left(s b_{1}-p c_{1}, \ldots, s b_{n}-p c_{n}\right)
$$

The lattice $L$ is completely determined by the rank two sublattice :

$$
\begin{gathered}
\tilde{L} \subset \operatorname{Ker}(\Phi):=\left\{(s, p) \in \mathbb{Z}^{\mathbf{2}} / s b_{i}-p c_{i} \equiv 0 \bmod a_{i}, \forall i=1, \ldots, n\right\} . \\
\tilde{L}=\left\{(s, p) \in \mathbb{Z}^{2} / s\left(\mathbf{a}_{\mathbf{1}}, \mathbf{h}_{\mathbf{n}+\mathbf{1}}\right)-p\left(\mathbf{a}_{\mathbf{2}}, \mathbf{h}_{\mathbf{n}+\mathbf{2}}\right) \in \mathbb{Z}\left(\mathbf{d}_{\mathbf{1}}, \mathbf{h}_{\mathbf{1}}\right)+\ldots+\mathbb{Z}\left(\mathbf{d}_{\mathbf{n}}, \mathbf{h}_{\mathbf{n}}\right)\right\}
\end{gathered}
$$


Remark 2 To any vector $(s, p) \in \tilde{L}$ with $s \geq 0$ we associate a unique binomial $B_{(s, p)} \in I_{L}$ in the following way: for any $i=1, \ldots, n$, let $v_{i}$ be the unique integer such that $s b_{i}-p c_{i}=v_{i} a_{i}$. We define the vectors $\mathbf{v}_{+}, \mathbf{v}_{-} \in \mathbb{I}^{n}$ by $\mathbf{v}_{+, i}=\max \left\{v_{i}, 0\right\}, \mathbf{v}_{-, i}=\max \left\{-v_{i}, 0\right\}$ and we must distinguish two cases:

- i) if $s \geq 0$ and $p \geq 0$ then $B_{(s, p)}=z^{s} \underline{x}^{\mathbf{v}_{-}}-y^{p} \underline{x}^{\mathbf{v}_{+}}$,

- ii) If $s \geq 0$ and $p<0$ then $B_{(s, p)}=z^{s} y^{-p} \underline{x}^{\mathbf{v}_{-}}-\underline{x}^{\mathbf{v}_{+}}$.

Let $D_{i}$ be the line $D_{i}=\left\{(s, p) \in \mathbb{R}^{2} \quad \mid s b_{i}-p c_{i}=0\right\}$. From now on, we suppose that the variables $x_{1}, \ldots, x_{n}$ are indexed in such a way that the slopes of the lines $D_{i}$ are in increasing order.

Lemma 3 Consider $B=M_{1}-M_{2} \in I_{L}$ a binomial, $M_{1}, M_{2}$ without common factors. We can write $B$ in only one of the followings forms:

1. $z^{s}-y^{p} x_{1}^{v_{1}} \ldots x_{n}^{v_{n}}, s>0 v_{i} \geq 0 \forall i$.

2. $y^{p}-z^{s} x_{1}^{v_{1}} \ldots x_{n}^{v_{n}}, p>0, v_{i} \geq 0 \forall i$.

3. $y^{p} z^{s}-x_{1}^{v_{1}} \ldots x_{n}^{v_{n}}, p, s>0 v_{i}>0 \forall i$.

4. $z^{s} x_{1}^{v_{1}} \ldots x_{k}^{v_{k}}-y^{p} x_{k+1}^{v_{k+1}} \ldots x_{n}^{v_{n}}, v_{i} \geq 0, p, s>0$, and $\exists 1 \leq i_{1} \leq k, k+1 \leq i_{2} \leq n / v_{i_{1}}, v_{i_{2}} \neq 0$. In other words if $(\mathbf{v}, s, p) \in L$, with $s, p>0$, and $\mathbf{v}=\left(v_{1}, \ldots, v_{n}\right)$ there exist $k$ such that $v_{i}<0$ for all $i<k$ and $v_{i} \geq 0$ for all $i \geq k$.

As a consequence we have the following lemma:

Lemma 4 1) There is no non trivial binomial in $I_{L}$, of the type: $z^{s} y^{p} \underline{x}^{\mathbf{v}}-\underline{x}^{\mathbf{v}_{+}}$with $s \geq 0, p \geq 0$, and $\mathbf{v}_{-} \neq 0$.

2) Consider an equality (where every fraction is reduced):

$$
\frac{z^{s} P_{1}(\underline{x})}{\underline{x}^{\mathbf{v}}}=\frac{y^{p} P_{2}(\underline{x})}{\underline{x}^{\mathbf{w}}} .
$$

- If there exist an index $i_{1}$ such that $v_{i_{1}}>0, w_{i_{1}}=0$, then for all $j \geq i_{1}, v_{j}>w_{j}$.

- If there exist an index $i_{2}$ such that $v_{i_{2}}=0, w_{i_{2}}>0$, then for all $j \leq i_{2}, v_{j}<w_{j}$.

The following proposition is an extension of [8], to the lattice case.

Proposition 1 We can describe a fan decomposition of $\mathbf{R}_{+}^{2}$, more precisely we have vectors $\varepsilon_{-1}, \varepsilon_{0}, \ldots, \varepsilon_{m+1} \in$ $\tilde{L} \cap \mathbf{Z}_{+}^{2}$ such that

- $\varepsilon_{-1}=\left(s_{-1}, 0\right), \varepsilon_{0}=\left(s_{0}, p_{0}\right)$, with $0 \leq s_{0} \leq s_{-1}$.

- Consider the Euclidean algorithm to compute the $\operatorname{gcd}\left(s_{-1}, s_{0}\right)$ :

$$
\begin{aligned}
s_{-1} & =q_{1} s_{0}-s_{1} \\
s_{0} & =q_{2} s_{1}-s_{2} \\
& \cdots \\
s_{m-1} & =q_{m+1} s_{m} \\
s_{m+1} & =0 \\
q_{i} \geq 2 & , s_{i} \geq 0 \quad \forall i
\end{aligned}
$$

Let $p_{i}$ be the sequence of integers defined by

$$
p_{i+2}=q_{i+2} p_{i+1}-p_{i} \quad-1 \leq i \leq m-1
$$

then $\varepsilon_{i}=\left(s_{i}, p_{i}\right)$. 
- $\varepsilon_{i}, \varepsilon_{i+1}$ is a basis of $\tilde{L}$ and $\operatorname{det}\left(\varepsilon_{i}, \varepsilon_{i+1}\right)=p_{0} s_{-1}>0$.

Note that the existence of the basis $\varepsilon_{-1}, \varepsilon_{0}$ is provided by [2], page 62 .

Definition 2 Let $r_{j, i}$ be the sequence of integers defined by

$$
r_{j, i}=\left(s_{i} b_{j}-p_{i} c_{j}\right) / a_{j} \quad-1 \leq i \leq m+1,1 \leq j \leq n
$$

and $\mathbf{r}_{i}$ the vector with coordinates $r_{j, i}$.

Lemma 5 1) Any of the sequences $s_{i}, p_{i}, r_{j, i}, 1 \leq j \leq n$ satisfy the recurrent relation:

$$
v_{i+2}=q_{i+2} v_{i+1}-v_{i} \text { for }-1 \leq i \leq m-1 .
$$

2) The sequences $s_{i}, r_{j, i}$ (for all $j$ ) are strictly decreasing but the sequence $p_{i}$ is strictly increasing.

3) Set $\nu\left(\right.$ resp. $\mu$ ) the greatest integer $j$ such that $\mathbf{r}_{j}=\mathbf{r}_{j,+}$ (resp. the smallest integer $j$ such that $\left.\mathbf{r}_{j}=-\mathbf{r}_{j,-}\right)$, then $-1 \leq \nu \leq \mu \leq m$.

4) $\operatorname{supp} \mathbf{r}_{i+1,+} \subset \operatorname{supp} \mathbf{r}_{i,+}$.

Theorem 7 1) The ring $R / I_{L}$ is arithmetically Cohen-Macaulay if and only if $\mu=\nu$. In this case the ideal $I_{L}$ is generated by:

$$
\begin{aligned}
F & =z^{s_{\nu}}-y^{p_{\nu}} x^{\mathbf{r}_{\nu}} \\
G & =y^{p_{\nu+1}}-z^{s_{\nu+1}} \underline{x}^{-\mathbf{r}_{\nu+1}} \\
H & =z^{s_{\nu}-s_{\nu+1}} y^{p_{\nu+1}-p_{\nu}} \underline{x}^{\mathbf{r}_{\nu}-\mathbf{r}_{\nu+1}}
\end{aligned}
$$

2) If $R / I_{L}$ is not arithmetically Cohen-Macaulay the ideal $I_{L}$ is generated by $\tau:=3+\left(q_{\nu+2}-\right.$ $1)+\ldots+\left(q_{\mu+1}-1\right)$ equations:

$$
\begin{aligned}
& \begin{aligned}
z^{s_{\nu}} & -y^{p_{\nu}} \underline{x}^{\mathbf{r}_{\nu}} \\
y^{p_{\nu+1}-p_{\nu}} z^{s_{\nu}-s_{\nu+1}} & -\underline{x}^{\mathbf{r}_{\nu}-\mathbf{r}_{\nu+1}}
\end{aligned} \\
& z^{s_{\nu+1}} \underline{x}^{\mathbf{r}_{\nu+1,-}}-{ }_{y} p_{\nu+1} \underline{x}^{\mathbf{r}_{\nu+1,+}} \\
& y^{2 p_{\nu+1}-p_{\nu}} z^{s_{\nu}-2 s_{\nu+1}}-\underline{x}^{\mathbf{r}_{\nu}-2 \mathbf{r}_{\nu+1}}
\end{aligned}
$$

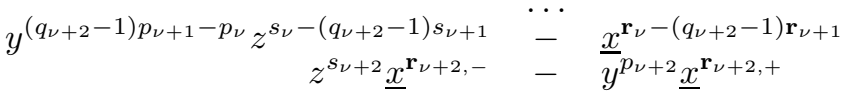

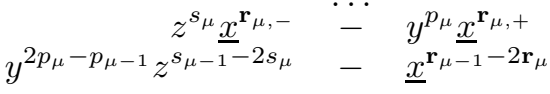

$$
\begin{aligned}
& \begin{aligned}
y^{\left(q_{\mu+1}-1\right) p_{\mu}-p_{\mu-1}} z^{s_{\mu-1}-\left(q_{\mu+1}-1\right) s_{\mu}} & \cdots \\
z^{s_{\mu+1}} \underline{x}^{\mathbf{r}_{\mu+1,-}} & -\underline{x}^{\mathbf{r}_{\mu-1}-\left(q_{\mu+1}-1\right) \mathbf{r}_{\mu}}
\end{aligned}
\end{aligned}
$$

They form a Groebner's basis for the reverse lexicographic order with respect to $z<y<x_{1}<$ $\ldots<x_{n}$. 
Proof .- Note that the proof given in [8], pp.1089, applies here without restriction. We outline the proof of 2): it consist to prove that the leading term of any binomial in $I_{L}$ for the reverse lexicographic order with respect to $z<y<x_{1}<\ldots<x_{n}$ is a factor of the leading term of some binomial in the above list. For example, let $B$ be a binomial corresponding to the lattice point $(\mathbf{v}, s, p)$ with $p \geq 0, s \geq 0$. By the fan decomposition of $\mathbb{R}_{+} \times \mathbb{R}_{+}$, there exists some $i \geq-1$ such that $(p, s)=\alpha \varepsilon_{i}+\beta \varepsilon_{i+1}$, with intehers $\alpha>0, \beta \geq 0$, this imply $\mathbf{v}=\alpha \mathbf{r}_{i}+\beta \mathbf{r}_{i+1}$. We need to consider three cases:

- if $i<\nu$ then the coordinates of $\mathbf{r}_{i}, \mathbf{r}_{i+1}$ are all positive, so the leading term of $B$ is $z^{s}$ but $s=\alpha s_{i}+\beta s_{i+1} \geq s_{\nu}$.

- By a similar argument if $i \geq \mu+1$ then the coordinates of $\mathbf{r}_{i}, \mathbf{r}_{i+1}$ are all negative, so the leading term of $B$ is $y^{p}$ but $p=\alpha p_{i}+\beta p_{i+1} \geq p_{\mu+1}$.

- if $\nu \leq i \leq \mu$ then the leading term of $B$ is $z^{s} \underline{x}^{\mathbf{v}-}$ which is a factor of $z^{s_{i}} \underline{x}^{\mathbf{r}_{i,-}}$.

If $B$ is a binomial corresponding to the lattice point $(\mathbf{v}, s, p)$ with $p<0, s \geq 0$. We argue with similar arguments using the fan decomposition of $\mathbb{R}_{+} \times \mathbb{R}_{-}$(that is every two consecutive vectors is a basis of $\tilde{L}$ ), given by the sequence of vectors

$$
\begin{gathered}
\varepsilon_{-1}-\varepsilon_{0}, \ldots, \varepsilon_{-1}-\left(q_{1}-1\right) \varepsilon_{0}=\varepsilon_{0}-\varepsilon_{1}, \ldots, \varepsilon_{0}-\left(q_{2}-1\right) \varepsilon_{1}=\varepsilon_{1}-\varepsilon_{2}, \ldots, \\
\varepsilon_{m-1}-\left(q_{m}-1\right) \varepsilon_{m}=\varepsilon_{m}-\varepsilon_{m+1},-\varepsilon_{m+1} .
\end{gathered}
$$

\section{Macaulayfication of codimension two simplicial toric rings}

The aim of this section consist to give an explicit description of the semigroup $S^{\prime}$ such that $K\left[S^{\prime}\right]$ is the Macaulayfication of the simplicial semigroup ring of codimension two $K[S]$. (see Theorem 5):

We recall that $S^{\prime}=\cap_{i=1}^{l}\left(S-\left(S \backslash P_{i}\right)\right)$ is a subsemigroup of $G(S)$, where $S \backslash P_{i}$ consist of the elements in $S$, which the $i$ coordinate is 0 . the ring

$$
K\left[S^{\prime}\right]=\cap_{i=1}^{l} K[S]_{\left(p_{\{i\}}\right)},
$$

is a Cohen-Macaulay ring, where $K[S]_{\left(p_{\{i\}}\right)}$ is a homogeneous localization and the intersection is in the localization $T^{-1} K[S]$, where $T$ is the set of all pure monomials. Remark that since $I_{L}$ is a lattice ideal any monomial is a non zero divisor for $K[S]$. Any simplicial group is trivially standard. In what follows we will write $I$ instead of $I_{L}$.

Remark 3 Since $S$ is simplicial of codimension two, every element in $S-\left(S \backslash P_{i}\right)$ can be viewed as a quotient of monomials $\frac{M(y, z, \underline{x})}{N(y, z, \underline{x})}$ where $M, N$ are monomials with disjoints supports and $N \notin p_{\{i\}}$, we notice that

$$
p_{\{i\}}= \begin{cases}\left(x_{i}, y, z\right) & \text { if } b_{i} \neq 0 \text { and } c_{i} \neq 0 \\ \left(x_{i}, y\right) & \text { if } b_{i}=0 \text { and } c_{i} \neq 0 \\ \left(x_{i}, z\right) & \text { if } b_{i} \neq 0 \text { and } c_{i}=0\end{cases}
$$

Lemma 6 Let $E \in \cap_{i=1}^{n}\left(S-\left(S \backslash P_{i}\right)\right)$, then for each $i$ we can write $E=\frac{z^{\alpha_{i}} y^{\beta_{i}} P_{i}(\underline{x})}{Q_{i}(\underline{x})}$, such that $x_{i}$ is not in the support of $Q_{i}$. 
Proof .- The assertion is clear if $b_{i} \neq 0$ and $c_{i} \neq 0$ for all $i=1, \ldots, n$. If $b_{i}=0$ and $c_{i} \neq 0$ then $s_{j} b_{i}-p_{j} c_{i}=-p_{j} c_{i} \leq 0$ and we have equality if and only if $i=-1$, this implies $\nu=-1, p_{\nu}=0$. Regarding the order introduced in the variables $x_{1}, \ldots, x_{n}$ by the lemma 3 , we can suppose that there exist natural integers $k, l$ such that $b_{1}=\ldots, b_{k}=0, a_{n-l}=\ldots a_{n}=0$ and that $k, l$ are the biggest possible. It will be enough to prove the Lemma for $i \leq k$ and $0<k<n$. Let $E \in \cap_{i=1}^{n}\left(S-\left(S \backslash P_{i}\right)\right), E=\frac{y^{\beta_{i}} P_{i}(\underline{x})}{Q_{i}(\underline{x}) z^{\alpha_{i}}}$ where $x_{i}$ is not in the support of $Q_{i}$, and $\alpha_{i}>0$. On the other hand for any $k<j<n-l$ we have $E=\frac{z^{\alpha_{j}} y^{\beta_{j}} P_{j}(\underline{x})}{Q_{j}(\underline{x})}$, where $x_{j}$ is not in the support of $Q_{j}$ remark that $x_{i}$ belongs to the support of $Q_{j}$, otherwise we have finish our proof, we can also assume that $P_{j}$ and $Q_{j}$ have disjoint support, this gives us the following element in $I$ :

$$
y^{\beta_{i}} P_{i}(\underline{x}) Q_{j}(\underline{x})-z^{\alpha_{j}+\alpha_{i}} y^{\beta_{j}} P_{j}(\underline{x}) Q_{i}(\underline{x})
$$

Since $x_{i}$ appears in the left side of this equality but no in the right side, we must have $\beta_{j}>\beta_{i}$. More precisely we write $Q_{j}(\underline{x})=x_{i}^{\gamma_{i}} \tilde{Q}_{j}(\underline{x}), P_{i}(\underline{x})=x_{i}^{\delta_{i}} \tilde{P}_{i}(\underline{x})$, and since the couple $\left(\alpha_{i}+\alpha_{j}, \beta_{j}-\beta_{i}\right)$ belongs to the lattice $\tilde{L}$, there exist integers $A, B$ such that:

$$
\left(\alpha_{i}+\alpha_{j}, \beta_{j}-\beta_{i}\right)=A\left(s_{-1}, 0\right)+B\left(s_{0}, p_{0}\right)
$$

this implies that $\beta_{j}-\beta_{i}=B p_{0}$. We have the following elements in $I$

$$
z^{s_{0}} \underline{x}^{\mathbf{r}_{0,-}-y^{p_{0}}} \underline{x}^{\mathbf{r}_{0,+}}, z^{B s_{0}} \underline{x}^{B \mathbf{r}_{0,-}}-y^{B p_{0}} \underline{x}^{B \mathbf{r}_{0,+}}
$$

this implies $\gamma_{i}+\delta_{i}=B \mathbf{r}_{0,-, i}$, and we have the following equality:

$$
\frac{z^{B s_{0}} \underline{x}^{B \hat{\mathbf{r}}_{0,-} x^{\delta_{i}}}}{\underline{x}^{B \mathbf{r}_{0,+}}}=\frac{y^{B p_{0}}}{x_{i} \gamma_{i}}=\frac{y^{\beta_{j}-\beta_{i}}}{x_{i} \gamma_{i}}
$$

where we have set $\mathbf{r}_{0,-, i}$ for the $i$-coordinate of the vector $\mathbf{r}_{0,-}$ and $\hat{\mathbf{r}}_{0,-}$ is the vector $\mathbf{r}_{0,-}$ with the $i$-coordinate equal to zero. Finally we have

$$
E=\frac{z^{\alpha_{j}} y^{\beta_{i}} P_{i}(\underline{x})}{\tilde{Q}_{j}(\underline{x})} \times \frac{z^{B s_{0}} \underline{x}^{B \hat{\mathbf{r}}_{0,-}} x^{\delta_{i}}}{\underline{x}^{B \mathbf{r}_{0,+}}}
$$

and $x_{i}$ is not in the support of the denominator, and we are done.

Lemma 7 Let $E \in \cap_{i=1}^{n}\left(S-\left(S \backslash P_{i}\right)\right)$. For each $i$ we write $E=\frac{z^{\alpha_{i}} y^{\beta_{i}} P_{i}(\underline{x})}{Q_{i}(\underline{x})}$, where $x_{i}$ is not in the support of $Q_{i}$, and we can assume that $P_{i}$ and $Q_{i}$ have disjoint support. Then:

1. For all $i$, we can assume that $\alpha_{i}<s_{\nu}$ and $\beta_{i}<p_{\mu+1}$. The equality $\frac{y^{\beta_{i}} P_{i}(\underline{x})}{Q_{i}(\underline{x})}=\frac{y^{\beta_{j}} P_{j}(\underline{x})}{Q_{j}(\underline{x})}$, such that $x_{i}$ is not in the support of $Q_{i}$ and $x_{j}$ is not in the support of $Q_{j}$, implies $i=\bar{j}$ and this equality is an identity. The same is true for $z$.

2. If there exists some index $i$ such that $\alpha_{i}=\beta_{i}=0$ then $E \in S$.

3. We can write $E=z^{\alpha} y^{\beta} E^{\prime}$ where $E^{\prime} \in S^{\prime}$, where $\alpha$ is the minimum of all the $\alpha_{i}$ and $\beta$ is the minimum of all the $\beta_{i}$. In particular we can assume that there exist indexes $i, j$ such that $\alpha_{i}=0$ and $\beta_{j}=0$. 
4. If $E=\frac{z^{\alpha} P(\underline{x})}{Q(\underline{x})}$, where $P$ and $Q$ have disjoint support, then $x_{1}$ is not in the support of $Q$.

Proof .-

1. Suppose that $\frac{y^{\beta_{i}} P_{i}(\underline{x})}{Q_{i}(\underline{x})}=\frac{y^{\beta_{j}} P_{j}(\underline{x})}{Q_{j}(\underline{x})}$ such that $x_{i}$ is not in the support of $Q_{i}, x_{j}$ is not in the support of $Q_{j}, i \neq j$ and $\beta_{j} \geq \beta_{i}$. It follows that $\tilde{P}_{i}(\underline{x}) \tilde{Q}_{j}(\underline{x})-y^{\beta_{j}-\beta_{i}} \tilde{P}_{j}(\underline{x}) \tilde{Q}_{i}(\underline{x})$ belongs to $I$ where $\tilde{P}_{l}=P_{l} / \operatorname{gcd}\left(P_{i}, P_{j}\right), \tilde{Q}_{l}=Q_{l} / \operatorname{gcd}\left(Q_{i}, Q_{j}\right)$. Since $0 \leq \beta_{j}-\beta_{i}<p_{\mu+1}$ such element cannot exists in $I$, and we are done.

2. Suppose that $E=\frac{P_{i}(\underline{x})}{Q_{i}(\underline{x})}$, such that $x_{i}$ is not in the support of $Q_{i}$ but $Q_{i} \neq 1$ and $P_{i}, Q_{i}$ have disjoint support. Let $x_{j}$ be in the support of $Q_{i}$, then we can write $E=\frac{z^{\alpha_{j}} y^{\beta_{j}} P_{j}(\underline{x})}{Q_{j}(\underline{x})}$, such that $x_{j}$ is not in the support of $Q_{j}$. It follows that $z^{\alpha_{j}} y^{\beta_{j}} P_{j}(\underline{x}) Q_{i}(\underline{x})-P_{i}(\underline{x}) Q_{j}(\underline{x})$ belongs to $I$. We get a contradiction since $x_{j}$ is not in the support of $P_{i} Q_{j}$.

3. It is clear that $\frac{z^{\alpha_{i}-\alpha} y^{\beta_{i}-\beta} P_{i}(\underline{x})}{Q_{i}(\underline{x})}=\frac{z^{\alpha_{j}-\alpha} y^{\beta_{j}-\beta} P_{j}(\underline{x})}{Q_{j}(\underline{x})}$ in the field of fractions of $K[S]$. We set $E^{\prime}=\frac{z^{\alpha_{i}-\alpha} y^{\beta_{i}-\beta} P_{i}(\underline{x})}{Q_{i}(\underline{x})}$, now it is clear that $E^{\prime} \in S^{\prime}$ and $E=z^{\alpha} y^{\beta} E^{\prime}$.

4. Suppose that $x_{1}$ is in in the support of $Q$, then we can write $\frac{z^{\alpha} P(\underline{x})}{Q(\underline{x})}=\frac{z^{\alpha_{1}} y^{\beta_{1}} P_{1}(\underline{x})}{Q_{1}(\underline{x})}$, such that $x_{1}$ is not in the support of $Q_{1}$ and $\beta_{1}>0$. It follows then that $z^{\alpha} P(\underline{x}) Q_{1}(\underline{x})-z^{\alpha_{1}} y^{\beta_{1}} P_{1}(\underline{x}) Q(\underline{x}) \in I$, but lemma 4, implies that $\alpha>\alpha_{1}$, and we get that $z^{\alpha-\alpha_{1}} P(\underline{x}) Q_{1}(\underline{x})-y^{\beta_{1}} P_{1}(\underline{x}) Q(\underline{x}) \in I$, and $x_{1}$ is the support of $P_{1}(\underline{x}) Q(\underline{x})$ but not in the support of $P(\underline{x}) Q_{1}(\underline{x})$, applying again lemma 4 , we get a contradiction since $\alpha-\alpha_{1}<s_{\nu}$.

Theorem 8 1. Any element in the minimal basis in I of the type

$$
z^{s_{\nu+l}} \underline{x}^{\mathbf{r}_{\nu+l},-}-y^{p_{\nu+l}} \underline{x}^{\mathbf{r}_{\nu+l},+},
$$

for $1 \leq l \leq \mu-\nu$, gives rise to a non trivial element

$$
E_{l}=\frac{y^{p_{\nu+l}}}{\underline{x}^{\mathbf{r}_{\nu+l},-}}=\frac{z^{s_{\nu+l}}}{\underline{x}^{\mathbf{r}_{\nu+l},+}} \in S^{\prime} .
$$

2. Any element $E \in S^{\prime}$ which can be written as

$$
\frac{y^{\beta}}{\underline{x}^{\mathbf{v}_{-}}}=\frac{z^{\alpha}}{\underline{x}^{\mathbf{v}_{+}}}
$$

where $\mathbf{v}_{+}, \mathbf{v}_{-}$have disjoint support, belongs to the semigroup generated by $S$ and the elements $E_{l}$, for $1 \leq l \leq \mu-\nu$.

3. Any element $E \in S^{\prime}$ which can be written as

$$
\frac{\left.y^{\beta} P(\underline{x})\right)}{\underline{x}^{\mathbf{v}}-}=\frac{z^{\alpha} Q(\underline{x})}{\underline{x}^{\mathbf{v}}},
$$

where $\mathbf{v}_{+}, \mathbf{v}_{-}$have disjoint support, belongs to the semigroup generated by $S$ and the elements $E_{l}$ for $1 \leq l \leq \mu-\nu$. 


\section{Proof .-}

1. It is clear that $E_{l}=\frac{y^{p_{\nu+l}}}{\underline{x}^{\mathbf{r}_{\nu+l},-}} \frac{z^{s_{\nu+l}}}{\underline{x}^{\mathbf{r}_{\nu+l},+}} \in S^{\prime}$. We have $E_{l} \notin S$ since $p_{\nu+l}<p_{\mu+1}$.

2. Let $E \in S^{\prime}$ such that $\frac{y^{\beta}}{\underline{x}^{\mathbf{v}_{-}}}=\frac{z^{\alpha}}{\underline{x}^{\mathbf{v}_{+}}}$, where $\mathbf{v}_{+}, \mathbf{v}_{-}$have disjoint support. It follows that $y^{\beta} \underline{x}^{\mathbf{v}_{+}}-z^{\alpha} \underline{x}^{\mathbf{v}_{-}}$belongs to $I$ then $(\alpha, \beta) \in \operatorname{ker} \Phi$ and there exist positive integers $k, \lambda_{1}, \lambda_{2}$ such that

$$
(\alpha, \beta)=\lambda_{1}\left(s_{k}, p_{k}\right)+\lambda_{2}\left(s_{k+1}, p_{k+1}\right)
$$

and as consequence of this

$$
v_{j}=\lambda_{1} r_{j, k}+\lambda_{2} r_{j, k+1} \text { for all } 1 \leq j \leq n .
$$

We recall that if $r_{j, l}>0$ for some $j, l$ then $r_{m, l}>0$ for all $m>j$ and that supp $\mathbf{r}_{k+1,+} \subset$ supp $\mathbf{r}_{k,+}$. By lemma 3, there exist $\delta$ such that $v_{l}<0$ for all $l<\delta$ and $v_{l} \geq 0$ for all $l \geq \delta$. Let supp $\mathbf{r}_{k,+}=\left\{l_{1}, \ldots, n\right\}$, supp $\mathbf{r}_{k+1,+}=\left\{l_{2}, \ldots, n\right\}$, with $l_{1} \leq l_{2}$. It follows that $l_{1} \leq \delta \leq l_{2}$ then we can write

$$
E=\frac{z^{\alpha}}{\underline{x}^{\mathbf{v}+}}=\left(\frac{x_{l_{1}}^{r_{l_{1}, k}} \ldots x_{\delta-1}^{r_{\delta-1, k}} z^{s_{k}}}{\underline{x}^{r_{k},+}}\right)^{\lambda_{1}}\left(\frac{x_{\delta}^{-r_{\delta, k+1}} \ldots x_{l_{2}-1}^{-r_{l_{2}-1, k+1}} z^{s_{k+1}}}{\underline{x}^{r_{k+1},+}}\right)^{\lambda_{2}}
$$

so

$$
E=\left(x_{l_{1}}^{r_{l_{1}, k}} \ldots x_{\delta-1}^{r_{\delta-1, k}} x_{\delta}^{-r_{\delta, k+1}} \ldots x_{l_{2}-1}^{-r_{l_{2}-1, k+1}}\right)^{\lambda_{2}} E_{k}^{\lambda_{1}} E_{k+1}^{\lambda_{2}} .
$$

3. If $E=\frac{y^{\beta} P(\underline{x})}{\underline{x}^{\mathbf{v}_{-}}}=\frac{z^{\alpha} Q(\underline{x})}{\underline{x}^{\mathbf{v}_{+}}}$, where $\mathbf{v}_{+}, \mathbf{v}_{-}$have disjoint support, then after division by the common factor of $P, \bar{Q}$ we can assume that they have disjoint support. But then $P(\underline{x}) \underline{x}^{\mathbf{v}}+$ and $Q(\underline{x}) \underline{x}^{\mathbf{v}-}$ have disjoint support. It follows that the element $E^{\prime}:=\frac{y^{\beta}}{Q(\underline{x}) \underline{x}^{\mathbf{v}_{-}}}=\frac{z^{\alpha}}{P(\underline{x}) \underline{x}^{\mathbf{v}_{+}}}$ belongs to $S^{\prime}$ and we can write $E=P(\underline{x}) Q(\underline{x}) E^{\prime}$, the assertion follows from the previous item.

Theorem 9 Any element $E \in S^{\prime}$ belongs to the semigroup generated by $S$ and the elements $E_{l}$ for $1 \leq l \leq \mu-\nu$.

Proof .- Let $E \in S^{\prime}$ be a non trivial element, by lemma 7 , item 4 we can write $E=\frac{z^{\alpha} P_{1}(\underline{x})}{x_{1}^{a_{1}^{1}} \ldots x_{n}^{a_{n}^{1}}}$ with $a_{1}^{1}=0$. Let $i_{1}$ be the biggest integer such that $a_{j}^{1}=0$ for $j<i_{1}$ but $a_{i_{1}}^{1}>0$. Since $E \in S^{\prime}=\cap_{i=1}^{n}\left(S-\left(S \backslash P_{i}\right)\right)$ we can write $E=\frac{y^{\beta_{1} z^{\alpha_{1}} P_{3}(\underline{x})}}{x_{1}^{a_{1}^{3}} \ldots x_{n}^{a_{n}^{3}}}$ with $a_{i_{1}}^{3}=0$ and $\beta_{1}>0$. It then follows that

$$
z^{\alpha} P_{1}(\underline{x}) x_{1}^{a_{1}^{3}} \ldots x_{n}^{a_{n}^{3}}-y^{\beta_{1}} z^{\alpha_{1}} P_{3}(\underline{x}) x_{1}^{a_{1}^{1}} \ldots x_{n}^{a_{n}^{1}} \in I,
$$

and lemma 4 implies that $0<\alpha_{1}<\alpha$, so we have that

$$
z^{\alpha-\alpha_{1}} P_{1}(\underline{x}) x_{1}^{a_{1}^{3}} \ldots x_{n}^{a_{n}^{3}}-y^{\beta_{1}} P_{3}(\underline{x}) x_{1}^{a_{1}^{1}} \ldots x_{n}^{a_{n}^{1}} \in I .
$$

Since $a_{i_{1}}^{1}>0, a_{i_{1}}^{3}=0$ then for all $j \geq i_{1}, a_{j}^{1} \geq a_{j}^{3}$ by lemma 4 . 
Thus we can write the equality:

$$
\frac{z^{\alpha-\alpha_{1}} P_{1}(\underline{x})}{x_{1}^{a_{1}^{1}} \ldots x_{i_{1}}^{a_{i_{1}}^{1}} x_{i_{1}+1}^{a_{i_{1}+1}^{1}-a_{i_{1}+1}^{3}} \ldots x_{n}^{a_{n}^{1}-a_{n}^{3}}}=\frac{y^{\beta_{1}} P_{3}(\underline{x})}{x_{1}^{a_{1}^{3}} \ldots x_{k_{1}-1}^{a_{k_{1}-1}^{3}}}
$$

Since the denominators have disjoint support, this equality gives one element $F_{1} \in S^{\prime}$ that belongs to the semigroup generated by $S$ and $E_{1}, \ldots, E_{\mu-\nu}$. Then we have that:

$$
E=F_{1} \frac{z^{\alpha_{1}}}{x_{i_{1}+1}^{a_{i_{1}+1}^{3}} \ldots x_{n}^{a_{n}^{3}}}
$$

Now either $a_{j}^{3}=0$ for all $j>i_{1}$, and in this case we have finished the proof of the theorem, or there exist $i_{2}>i_{1}$ such that $a_{j}^{3}=0$ for all $i_{1} \leq j<i_{2}$, but $a_{i_{2}}^{3}>0$. Since $E \in S^{\prime}=\cap_{i=1}^{n}\left(S-\left(S \backslash P_{i}\right)\right)$ we can write $E=\frac{y^{\beta_{2}} z^{\alpha_{2}} P_{4}(\underline{x})}{x_{1}^{a_{1}^{4}} \ldots x_{n}^{a_{n}^{4}}}$ with $a_{i_{2}}^{4}=0$ and $\beta_{2} \geq 0$. We have the following element in $I_{L}$

$$
y^{\beta_{2}} z^{\alpha_{2}} P_{4}(\underline{x}) \underline{x}^{\left(\mathbf{a}_{3}-\mathbf{a}_{4}\right)_{+}}-y^{\beta_{1}} z^{\alpha_{1}} P_{3}(\underline{x}) \underline{x}^{\left(\mathbf{a}_{3}-\mathbf{a}_{4}\right)-}
$$

First since $\alpha_{1}<s_{\nu}, \alpha_{2}<s_{\nu}, \beta_{1}<p_{\mu+1}, \beta_{2}<p_{\mu+1}$, we must have $\alpha_{1} \neq \alpha_{2}, \beta_{1} \neq \beta_{2}$.

Now suppose that $\beta_{2}<\beta_{1}$, we have two cases:

1. If $\alpha_{1}>\alpha_{2}$ then Lemma 4 , implies that $a_{i}^{3}>a_{i}^{4}$ for all $i$ but $a_{i_{1}}^{3}=0$, this is a contradiction.

2. If $\alpha_{1}<\alpha_{2}$ since $a_{i_{2}}^{3}>0, a_{i_{2}}^{4}=0$ by Lemma 4 , we get $a_{i}^{3}>a_{i}^{4}$ for all $i \leq i_{2}$ but $a_{i_{1}}^{3}=0$, this is a contradiction.

So we have $\beta_{2}>\beta_{1}$, if we assume that $\alpha_{1}<\alpha_{2}$ since $a_{i_{2}}^{3}>0, a_{i_{2}}^{4}=0$ by lemma 4 we have $a_{i}^{3}>a_{i}^{4}$ for all $i \leq i_{2}$ but $a_{i_{1}}^{3}=0$, this is a contradiction. Finally we get $\beta_{2}>\beta_{1}$ and $\alpha_{1}>\alpha_{2}$.

Using lemma 4 , we argue as before and we get that for all $j \geq i_{2}, a_{j}^{3} \geq a_{j}^{4}$. We have the following equality:

$$
\frac{z^{\alpha_{1}-\alpha_{2}}}{x_{i_{2}}^{a_{i_{2}}^{3}-a_{i_{2}}^{4}} \ldots x_{n}^{a_{n}^{3}-a_{n}^{4}}}=\frac{y^{\beta_{2}-\beta_{1}} P_{4}(\underline{x})}{P_{3}(\underline{x}) x_{1}^{a_{1}^{4}} \ldots x_{i_{2}-1}^{a_{i_{2}-1}^{4}}} .
$$

This equality defines one element $F_{2} \in S^{\prime}$ that belongs to the semigroup generated by $S$ and $E_{1}, \ldots, E_{\mu-\nu}$, and we have

$$
E=F_{1} F_{2} \frac{z^{\alpha_{2}}}{x_{i_{2}+1}^{a_{i_{2}+1}^{4}} \ldots x_{n}^{a_{n}^{4}}}
$$

We can continue and we can write

$$
E=F_{1} F_{2} \ldots F_{m},
$$

where $F_{1}, F_{2}, \ldots, F_{m}$ belong to the semigroup generated by $S$ and $E_{1}, \ldots, E_{\mu-\nu}$. This ends the proof of the theorem.

Example 7 Let $k$ be a non zero natural number, and consider the simplicial toric variety defined parametrically by:

$$
x_{1}=u_{1}^{2 k}, \ldots, x_{k}=u_{k}^{2 k}, y=u_{1}^{k+1} u_{2} u_{3} \ldots u_{k}, z=u_{1} u_{2}^{k+1} u_{3} \ldots u_{k}
$$

It is a codimension two variety in $\mathbb{P}^{k+1}$. Let $I_{k}$ be the vanishing ideal of this variety. We apply the algorithm described in proposition 1 to find a system of generators of $I_{k}$ : 


$$
\begin{aligned}
& y^{2 k}-x_{1}^{k+1} x_{2} x_{3} \ldots x_{k} \\
& z^{2} x_{1}-y^{2} x_{2} \\
& y^{2 k-2} z^{2}-x_{1}^{k} x_{2}^{2} x_{3} \ldots x_{k} \\
& y^{2 k-4} z^{4}-x_{1}^{k-1} x_{2}^{3} x_{3} \ldots x_{k} \\
& y^{2} z^{2 k-2} \stackrel{\cdots}{-} x_{1}^{2} x_{2}^{k} x_{3} \ldots x_{k} \\
& z^{2 k}-x_{1} x_{2}^{k+1} x_{3} \ldots x_{k}
\end{aligned}
$$

In order to get the Macaulayfication we must consider the element:

$$
\frac{y^{2}}{x_{1}}=\frac{z^{2}}{x_{2}}=u_{1}^{2} u_{2}^{2} u_{3}^{2} \ldots u_{k}^{2}
$$

The Macaulayfication will be the semigroup ring :

$$
K\left[S^{\prime}\right]=K\left[u_{1}^{2 k}, \ldots, u_{k}^{2 k}, u_{1}^{k+1} u_{2} u_{3} \ldots u_{k}, u_{1} u_{2}^{k+1} u_{3} \ldots u_{k}, u_{1}^{2} u_{2}^{2} u_{3}^{2} \ldots u_{k}^{2}\right] .
$$

In fact it is easy to check that

$$
K\left[S^{\prime}\right]=K\left[x_{1}, x_{2}, x_{3}, \ldots, x_{k}, y, z, w\right] /\left(z^{2}-x_{2} w, y^{2}-x_{1} w, w^{k}-x_{1} x_{2} x_{3} \ldots x_{k}\right),
$$

and it is a complete intersection.

Example 8 We can apply our methods to some non toric cases. The (non-toric) variety $V \subset \mathbb{A}^{7}$ defined by

$$
x_{1}=s^{4}+t^{4} ; x_{2}=s^{2} t u ; x_{3}=s^{3} t ; x_{4}=s t^{3} ; x_{5}=s u^{3} ; x_{6}=s^{2} t^{2} v ; x_{7}=v
$$

is a generalized f-variety, not locally Cohen-Macaulay, and $\operatorname{dim} V=4$.

Let $V_{1}$ be the variety defined by

$$
x_{1}=s^{4}+t^{4} ; x_{2}=s^{2} t u ; x_{3}=s^{3} t ; x_{4}=s t^{3} ; x_{5}=s u^{3} ; x_{6}=s^{2} t^{2} ; x_{7}=v .
$$

Let $K[V]$ and $K\left[V_{1}\right]$ be respectively the coordinate rings of $V$ and $V_{1}$. It is immediate to check that $V_{1}$ is a complete intersection, and therefore it is arithmetically Cohen-Macaulay, in fact $V_{1}$ is the Macaulayfication of $V$.

\section{References}

[1] N.T. Cuong. Remarks on the Non-Cohen-Macaulay locus of Noetherian schemes. Proc. Amer. Math. Soc. 126-4 (1998), 1017-1022.

[2] H. Cohn. A second course in number theory. New York and London: John Wiley and Sons, Inc. XIII, 276 p. (1962).

[3] D. Eisenbud and B. Sturmfels. Binomials ideals. Duke Math. J. 84-1 (1996), 1-45.

[4] S. Goto. Approximatively Cohen-Macaulay Rings. Journal of Algebra 76 (1982), 214-225. 
[5] S. Goto, N. Suzuki and K. Watanabe. On affine semigroups. Japan Journal Math. 2 (1976), $1-12$.

[6] M. Hochster. Rings of invariants of tori, Cohen-Macaulay rings generated by monomials, and polytopes. Ann. Math. 96, 318-337 (1972).

[7] T. Kawasaki. On Macaulayfication of Noetherian Schemes. Trans. AMS 352-6 (2000, 25172552.

[8] M. Morales. Equations des Variétés Monomiales en codimension deux. Journal of Algebra 175 (1995), 1082-1095.

[9] I. Peeva and B. Sturmfels. Syzygies of codimension 2 lattices ideals. Maths Z. 298-1 (1986), $145-167$.

[10] N.V. Trung and L.T. Hoa. Affine Semigroups and Cohen-Macaulay rings generated by monomials. Trans. AMS 298-1 (1986), 145-167.

[11] P. Schenzel. Dualisierende komplexe in der lokalen Algebra und Buchsbaum-Ringe. Lectures Notes in Maths 907 (1982), Springer-Verlag.

[12] P. Schenzel. On the use of local cohomology in Algebra and Geometry, in Six lectures on commutative Algebra, J. Elias et als editors. Progress in Maths (1998), Birkhauser.

[13] P. Schenzel. On birational Macaulayfications and Cohen-Macaulay canonical modules. $J$. Algebra 275, No.2, 751-770 (2004). 